\title{
Asimetrías entre profesores y estudiantes: disputas emocionales e ideológicas ante la formación de las élites
} \section{de Educación, N. ${ }^{\circ} 70$. Primer semestre de 2016, Bogotá, Colombia.}

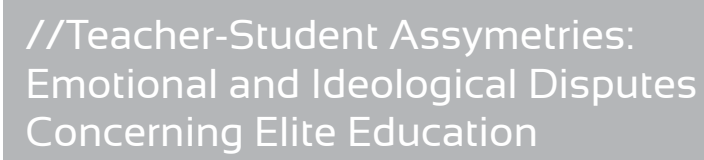

\section{//Assimetrias entre professores e estudantes: disputas emocionais e ideológicas ante a formação das elites}

Sandra Ziegler*

\begin{abstract}
Doctora en Ciencias Sociales de la Facultad Latinoamericana de Ciencias Sociales, Investigadora Senior del Programa Educación, Conocimiento y Sociedad, Area Educación, Facultad Latinoamericana de Ciencias Sociales. Sede Argentina. Correo electrónico: sziegler@flacso.org.ar
\end{abstract}

\section{Resumen}

El artículo analiza la labor emprendida por los profesores secundarios que tienen a su cargo la formación de las élites en Buenos Aires. Con tal fin, se revisan las adscripciones y las disputas emocionales e ideológicas de los docentes ante el ejercicio de su trabajo en las instituciones educativas. El estudio aborda las múltiples operaciones y el trabajo deliberado que desarrollan los profesores para generar asimetría ante sus estudiantes. Asimismo, se revisan las diferencias identificadas en escuelas formadoras de élites públicas y privadas por cuanto para el caso argentino existen ambas opciones de establecimientos que portan patrones de reclutamiento y una selección diferente de sus estudiantes.

Este trabajo integra los resultados de un estudio cualitativo realizado en cuatro escuelas, donde se entrevistaron treinta profesores de diferentes asignaturas. La elección de las escuelas combinó distintas variables: tres de ellas pertenecen al sector privado (una es confesional y las otras dos son laicas) y una es de dependencia pública, todas ellas se proclaman como instituciones formadoras de élites.

Los mecanismos que los profesores emplean para generar asimetría con respecto a sus estudiantes se conceptualizan en clave de las diferencias de los patrones de reclutamiento de cada tipo de escuelas, ya que estos últimos son altamente explicativos en relación con las posiciones diferenciales asumidas por los docentes. Observamos en estos casos que la asimetría resulta para ellos una condición central en los vínculos pedagógicos contemporáneos.

\section{Abstract}

This article tackles the issue of the work of high school teachers in elite education institutions in Buenos Aires. For this purpose, the study analyzes their positions and their own emotional and ideological disputes concerning their work in those institutions. The research addresses the diverse strategies developed in order to create asymmetries between them and their students.

At the same time, the differences between public and private elite schools are studied. Both models coexist in Argentina, each having different recruitment patterns and selection procedures.

This article gathers the results of a qualitative research based on interviews to thirty teachers from diverse subjects, from four different schools. Three of them are private (one religious, two secular), and the last one is public. All of them claim themselves as elite education institutions.

\section{Palabras clave}

Educación secundaria, profesores, asimetrías, élites, escuelas públicas y privadas

\section{Keywords}

Secondary school, teachers, asymmetry, elite, public and private schools

\section{Palavras chave}

Educação do ensino secundário, professores, assimetrias, escolas públicas e particulares 
The mechanisms developed by the teachers in order to establish that asymmetry vary in connection with the recruitment pattern of each of those elite schools. It is noticeable that asymmetry for them is a core condition of their pedagogical relationship with their students.

\section{Resumo}

O artigo analisa o trabalho empreendido pelos professores de ensino secundário que têm em sua responsabilidade a formação das elites em Buenos Aires. Com esse fim, revisam-se as adscrições e as disputas emocionais e ideológicas dos docentes frente ao exercício do seu trabalho nas instituições educativas. O estudo aborda as múltiplos operações e o trabalho deliberado que desenvolvem os professores para gerar assimetria frente a seus estudantes. Assim mesmo, revisam-se as diferenças identificadas nas escolas formadoras de elites públicas e particulares, enquanto para o caso argentino existem as duas opções de estabelecimentos que aportam patrões de recrutamento e uma seleção diferente de seus estudantes.

Neste trabalho integra os resultados de um estudo qualitativo realizado em quatro escolas, onde se entrevistaram trinta professores de diferentes assinaturas. A seleção das escolas combinou distintas variáveis: três delas pertencem ao setor privado (uma é confessional e as outras duas são laicas) e uma é de dependência pública, todas elas se aclamam como instituições formadoras de elites.

Os mecanismos que os professores empregam para gerar assimetria com respeito a seus estudantes, conceitualizam-se em clave das diferenças dos patrões de recrutamento de cada tipo de escola, já que esses últimos são altamente explicativos em relação com as posições diferenciais assumidos pelos docentes. Enxergamos nestes casos que a assimetria resulta para eles uma condição central nos vínculos pedagógicos contemporâneos.

\section{Introducción}

Este trabajo ${ }^{1}$ se sitúa en la confluencia de dos campos de estudio que habitualmente no se han explorado en conjunto: los procesos de formación de las élites (por la vía de la escolarización) y la indagación sobre los profesores de nivel secundario que tienen a su cargo la preparación de estos grupos. En particular, el artículo analiza la labor emprendida por estos profesores revisando sus adscripciones y disputas emocionales e ideológicas ante el ejercicio de su trabajo en las instituciones educativas.

Ante un escenario de fragmentación educativa (Kessler, 2002; Tiramonti, 2004), en el cual las aulas son internamente más homogéneas en relación con la composición de sus alumnos, es factible identificar profesores que educan a hijos de familias que detentan posiciones de poder (fuera de la escuela) y alumnos que fueron seleccionados por sus capacidades académicas (en

Agradezco a Nancy Montes por el aporte de los datos estadísticos acerca del estado de la educación privada en Argentina, y también a los evaluadores del presente artículo por brindarme sugerencias que han contribuido a mejorar la versión final. 
los colegios universitarios públicos de élite). En ese espacio escolar los docentes están compelidos a erigirse y ocupar una posición de asimetría en relación con sus estudiantes. Si bien crear tal asimetría es una condición indispensable para el ejercicio de la transmisión escolar, en los casos que indagaremos se presenta la particularidad de que los alumnos y, sobre todo, sus familias de origen poseen condiciones de estatus y prestigio (ya sea por sus ingresos económicos; apellidos; inserciones sociales, políticas, culturales, etc.) que conducen a que los profesores desarrollen algunas operaciones para lograr detentar una relación desigual y de poder en el espacio escolar. En síntesis, los profesores despliegan mecanismos con el fin de crear una condición asimétrica (Narodowski, 2014) ante sus alumnos que provienen de los circuitos más selectos (ya sea por la procedencia de sus familias o por sus condiciones académicas, como veremos más adelante).

Este trabajo indaga sobre las múltiples operaciones y el trabajo deliberado que desarrollan los profesores para generar una relación que les posibilite ser reconocidos como una autoridad pedagógica investida de legitimidad. Asimismo, se revisan las diferencias identificadas en escuelas formadoras de élite tanto públicas como privadas ya que para el caso argentino se advierte que ambas opciones de establecimientos presentan diferentes patrones de reclutamiento y selección de sus estudiantes: aquellos que se rigen bajo los criterios de la meritocracia (escuelas públicas y gratuitas dependientes de la universidad que representan una tendencia numéricamente muy minoritaria) y la vía que privilegia la posesión de recursos económicos como criterio excluyente para el acceso a los establecimientos (en las escuelas privadas orientadas a las familias de elevados ingresos).

Los procesos de elección escolar de las familias se encuentran imbricados con los patrones de reclutamiento que desarrollan las diferentes escuelas. De este modo, resulta factible observar que las familias presentan diferentes apuestas al seleccionar escuelas en el contexto de una sociedad en donde el acceso a las posiciones de élite así como su mantenimiento y legitimación están escasamente institucionalizados (Tiramonti \& Ziegler, 2008) y, por ende, exigen desarrollar estrategias activas para arribar y sostener las posiciones de élite. La informalidad y la disputa por el acceso a tales posiciones explica que las familias realicen sus opciones incorporando a sus hijos en diferentes escuelas según sus preferencias, perfiles socioculturales y económicos. De este modo, seleccionan establecimientos que resultan acordes a las estrategias que desarrollan en un contexto en donde los mecanismos de selección de las élites son difusos, se encuentran débilmente institucionalizados y los sujetos deben combinar múltiples recursos (simbólicos y materiales) para pujar por el acceso al universo de las élites. Ante esta dinámica es 
factible identificar un proceso de fragmentación entre las escuelas, en la medida en que estas constituyen espacios autorferidos que portan un universo cultural y social que les es propio y que se encuentra altamente deferenciadas de las restantes. En este escenario el presente trabajo aborda el trabajo que los profesores efectúan para entablar una relación asimétrica con los alumnos, que posibilite la transmisión pedagógica.

En primer lugar, el artículo ubica el espacio y donde se ha desarrollado el trabajo de campo, sus caraterísticas, así como algunos datos de contexto que resultan fudamentales para comprender los ámbitos donde se concentran las escuelas que procuran formar élites en Buenos Aires. En segundo lugar, se presentan las posiciones y operaciones que los profesores despliegan tanto en las escuelas privadas como en la institución pública indagada, procurando caracterizar y analizar los mecanismos para la insturación de las asimetrías en cada caso. Finalmente, dichas asimetrías se conceptualizan a la luz de las diferencias de los patrones de reclutamiento de cada tipo de escuelas, por cuanto estos últimos son altamente explicativos en relación con las posiciones diferenciales asumidas por los docentes. También en las conclusiones se brindan indicios acerca de la intencionalidad entre estos profesores de instaurar una relación pedagógica que los invista de legitimidad. Observamos en estos casos que para ellos la asimetría resulta una condición central en los vínculos pedagógicos contemporáneos.

\section{Metodología}

El presente trabajo se basa en un estudio realizado en escuelas secundarias $^{2}$ ubicadas en la Ciudad de Buenos Aires y en la zona norte del Conurbano Bonaerense ${ }^{3,4}$. En Argentina la formación orientada a los sectores de élite se imparte fuertemente en el sector de la educación privada. Si analizamos los alumnos que asisten

2 La escuela secundaria argentina comprende las edades teóricas de 13 a 17 años.

3 El mismo se ha realizado en el marco de la tesis doctoral Docentes de la élite, élites docentes: la configuración de la labor pedagógica y de una comunidad de profesores para la formación de los sectores privilegiados, dirigida por Guillermina Tiramonti y Marcelo Caruso. Doctorado en Ciencias Sociales (Flacso, Argentina), noviembre de 2011

4 Según datos del Censo Nacional de Población del año 2010, el Conurbano Bonaerense concentra más del $60 \%$ de la población de la Provincia de Buenos Aires. Se trata de una zona densamente poblada. En esta región reside casi el $25 \%$ de la población y el 24,6\% de los alumnos de todo el país. Por su parte, la Ciudad de Buenos Aires representa una de las metrópolis más prósperas a escala nacional y si bien su peso demográfico es del $7,2 \%$ de la población del país, es el espacio de concentración del poder político, cultural y económico. Allí se escolariza el 6,4\% de los alumnos del total nacional. 
a escuelas privadas sobre el total de la población escolar, las tasas más altas de privatización se ubican en los conglomerados más prósperos y centrales. Asimismo, dicho sector se encuentra en proceso de crecimiento en los últimos años ${ }^{5}$.

En el año 2014, frente a un promedio nacional de $29,1 \%$ de alumnos en escuelas privadas en todo el país, en la Ciudad de Buenos Aires el porcentaje asciende al $51 \%$ y en la Provincia de Buenos Aires es de un $35,7 \% \%^{6}$. En esta última, el nivel de privatización es más pronunciado en los municipios más ricos. A modo de ejemplo, en el año 2013, Vicente López (68\%) y San Isidro (64\%) representan las localidades dentro de la Provincia de Buenos Aires con porcentajes más elevados de alumnos en educación común en establecimientos privados ${ }^{7}$.

Además de la concentración geográfica, la composición socioeconómica de la matrícula privada también da cuenta de su creciente asociación con los sectores más prósperos. En el año 2011 en los hogares del Área Metropolitana (Ciudad de Buenos

5 A modo de ejemplo: en el año 2007 el porcentaje de alumnos en la educación común privada en el país ascendía a 27,3; en el año 2014 el $29,1 \%$ de los alumnos asiste a un establecimiento privado. Fuente: Relevamiento Anual, Diniece, Ministerio de Educación de la Nación.

6 Fuente: Elaboración propia con base en datos del Relevamiento anual 2014. DiNiece, Ministerio de Educación de la Nación.

7 La educación común abarca los niveles inicial, primario, secundario y superior no universitario. Fuente: elaboración propia con base en datos del Relevamiento anual 2013. Dirección de Información y Estadística. Dirección General de Cultura y Educación, Provincia de Buenos Aires.
Aires y sus suburbios) pertenecientes a la población que concentra el quinto quintil de ingresos, el $57 \%$ de los alumnos de nivel secundario asistían a escuelas privadas ${ }^{8}$. La educación secundaria pública que es gratuita y que se orienta a la formación de élites se encuentra representada solo por un grupo reducido de escuelas dependientes de la universidad y un número muy escaso de establecimientos de dependencia estatal no universitarios colonizados por las familias de los sectores más acomodados.

En síntesis, la concentración de la formación de las élites en el nivel de la educación secundaria en Argentina se produce en los espacios urbanos más prósperos y mayoritariamente en las escuelas del sector privado donde el acceso no es certificado inicialmente por la capacidad académica de los alumnos, sino por las posibilidades económicas de sus familias. Como hemos señalado, en el caso de la mayoría de las escuelas públicas universitarias, el acceso se dirime por criterios meritocráticos a través de la aplicación de un examen de ingreso excluyente. Esas instituciones son gratuitas.

Este trabajo integra los resultados obtenidos en un trabajo de campo desarrollado en cuatro escuelas secundarias, en donde se realizaron entrevistas en profundidad a treinta

8 Fuente: Elaboración propia con base en datos de la Encuesta Permanente de Hogares 2011, Instituto Nacional de Estadísticas y Censos. El quinto quintil de la población es la quinta parte de la población que concentra los ingresos económicos más altos. 
profesores de diferentes asignaturas y sus directivos. Se trata de instituciones de amplia trayectoria, reconocidas por su prestigio. La mayoría se define como instituciones "tradicionales" y de "excelencia académica". La elección de las escuelas combinó distintas variables. Tres de ellas pertenecen al sector privado, una es confesional y las otras dos son laicas (una bilingüe y la otra trilingüe). Se incluyó además una escuela pública cuyos mecanismos de selección en el ingreso y su trayectoria en la formación de la dirigencia nacional dan cuenta de su carácter de institución que ha formado a grupos de élite (básicamente de corte político e intelectual). En este trabajo, seleccionamos instituciones que explícitamente se proponen la preparación de sus estudiantes para el ejercicio de posiciones de poder y de privilegio. Por posiciones de privilegio entendemos la pertenencia a grupos favorecidos en los terrenos económico, social y cultural.

\section{Asimetrías y afectos docentes ante la escolarización en espacios de privilegio}

Como señalamos, en estas páginas indagaremos aspectos ligados a la afectividad de los profesores ante la situación que representa entablar una relación educador-educando con estudiantes pertenecientes a medios favorecidos o que formarán parte de las élites. En estos casos, advertimos que la tensión por generar relaciones asimétricas y la necesidad de imponer una autoridad reconocida está presente en las escuelas indagadas. Narodowski define la asimetría como:

[...] una relación en la cual al aplicar una regla o una operación, el resultado obtenido difiere en cada uno de los elementos de la relación. En el caso de la relación adultoniño/adolescente, ese resultado muestra propiedades significativamente diferenciadas entre unos y otros. Entre los términos de la relación asimétrica existe desigualdad aunque no necesariamente desequilibrio [...]. La asimetría adulto-niño implica que el vínculo entre uno y otro no es entre iguales, sino todo lo contrario. (2014, p. 197)

Si bien los profesores representan a la autoridad pedagógica en el escenario escolar, el vínculo está mediado por la distancia y la diferencia de poder en el espacio social que se puede reconocer entre ellos y las familias de procedencia de los alumnos. Así, si toda relación pedagógica presupone cierta asimetría que constituye una 
condición para el establecimiento de un vínculo pedagógico para hacer posible la transmisión cultural, dicha diferencia tiene la particularidad de producirse en un contexto en donde el poder del profesor se ejerce ante quienes se espera que sean los futuros poderosos, o ante los hijos de figuras que efectivamente detentan posiciones de poder en la sociedad actual.

En efecto, la asimetría ha sido una condición planteada como idea fuerza y vector que permitió el desarrollo de la escena escolar al calor de la modernidad. La condición asimétrica del vínculo pedagógico presenta un extenso tratamiento en el campo educativo; tanto la caracterización clásica de la educación como relación entabalada entre las generaciones adultas y jóvenes planteada por Durkheim en Educación y sociología, las nociones de autoridad pedagógica y el ejercicio de la violencia simbólica desarrolladas por Bourdieu y Passeron en La reproducción, así como los aportes acerca de las características propias de la configuración de la escuela moderna (Antelo, 2007; Dubet, 2006; Dussel \& Caruso, 1999 y Narodowski, 2014), denotan la presencia de la asimetría en los vínculos pedagógicos.

En nuestras entrevistas frecuentemente la asimetría ha surgido asociada a la necesidad de que los docentes se instauren como una figura de autoridad reconocida. Autores clásicos como Weber (1996) o más recientemente Kòjeve (2005) han dado cuenta de los procesos de dominación legítima. Kòjeve, en un sentido semejante a Weber, caracteriza la autoridad como "la posibilidad que tiene un agente de actuar sobre los demás (o sobre otro), sin que esos otros reaccionen en su contra, siendo totalmente capaces de hacerlo" (Kòjeve, 2005, p. 36). Así, cabe señalar que a partir de la formulación de Weber, Kòjeve plantea que la autoridad no implica el uso de la fuerza, por cuanto, si hay reacción o resistencia, la autoridad no es tal.

En este sentido, nos interesa aproximarnos a las disputas emocionales e ideológicas de los profesores, entendidas estas como las tensiones, contradicciones y distancias que señalan con relación a su labor de formar a este sector en particular. Entre otras preguntas posibles indagaremos: ¿Cuáles son los vínculos que entablan los docentes de estas instituciones con sus alumnos que provienen de un entorno diferente? ¿Qué sentimientos despierta educar a los hijos de los poderosos? ¿Cómo iniciden las instituciones y sus modos de reclutamiento en los vínculos entablados? ¿Hay diferencias según las instituciones y su carácter público o privado? ¿Cómo se manifiestan las relaciones de superioridad y subordinación entre profesores y estudiantes? ¿Cómo se desarrollan estos afectos? ¿Hay diferencias en los vínculos que entablan los profesores con los jóvenes y con las familias? ¿Qué identificaciones y qué distancias manifiestan los profesores con sus alumnos? ¿Qué sienten por sus alumnos y el trabajo que desempeñan? ¿Hay patrones comunes para el establecimiento de los vínculos con sus alumnos? 
Abrir interrogantes de este orden implica ingresar en la arena de la afectividad de estos profesores. No es el ánimo de este artículo realizar una lectura en clave psicológica de estos procesos, sino más bien abordar el terreno de los afectos en la labor de los docentes.

Abramovski (2010) refiere al afecto magisterial haciendo alusión a "la especificidad del afecto que los docentes sienten por sus alumnos" (2010, p. 23). Al dar cuenta de los afectos docentes, los explica como una construcción histórica y socialmente situada que circunscribe el universo de sentimientos posibles y reconoce una serie de prácticas afectivas docentes.

Si bien los estudios sobre la afectividad docente empiezan a adquirir un lugar en la investigación local de los últimos años, muchos de los abordajes desarrollados en nuestro país se centraron en las relaciones pedagógicas entre profesores y alumnos ante el crecimiento de la pobreza y su repercusión en el escenario escolar (Antelo, 2007; Duschatzky \& Redondo, 2000; Martinis \& Redondo, 2006; entre otros), y se registran escasos estudios locales (Kessler, 2002; Rodríguez Moyano, 2012) que se aproximen a las relaciones de los docentes con los alumnos que forman parte del grupo de los "poderosos" destacar el aporte de Rauscher (2010), quien ha tematizado el vínculo de los profesores formadores de las futuras élites y sus estudiantes. Dicho autor da cuenta de estos profesores como una élite (dentro del cuerpo de los profesores en general) al servicio de estos estudiantes, y caracteriza las relaciones de "devoción" y de atención personal que les dispensan a sus discipulos que formarán parte de las posiciones más encumbradas. A diferencia de nuestro trabajo, las relaciones identificadas por Rauscher se asocian a un sistema muy estructurado de formación de élites, en el cual los profesores se constituyen en "entrenadores" que preparan a sus estudiantes para el riguroso sistema de exámenes y concursos que deberán sortear para el futuro acceso a posiciones gravitantes. Por otra parte, algunos trabajos desarrollados en otros países abordan tangencialmente la relación entre profesores y estudiantes al dar cuenta de los procesos de formación de élites, pero no profundizan en el vínculo específico entre estos, sino

9 Ambos autores señalan las distancias entre las familias y escuelas que se advierten en instituciones que atienden a sectores altos; sin embargo, ninguno de los trabajos indaga sobre las relaciones pedagógicas entabladas entre los profesores y sus estudiates. 
más bien en las particularidades que tienen los propios estudiantes y los entornos en los que se socializan (Darmon, 2013 ; Daverne \& Dutercq, 2013; Kahn; 2011, entre otros).

En nuestro estudio, los entrevistados recurrentemente hacen señalamientos acerca de quiénes son sus alumnos y las familias de origen. "Acá los padres son los dueños de la mitad del país", menciona una profesora de Historia del Arte de la escuela bilingüe. Otra profesora de Historia del colegio trilingüe plantea:

[los padres] son gente de un poder adquisitivo muy alto, muy acostumbradas a manejarse e imponer ellos sus tiempos y demás. Tienen esas características. Muchas veces se presentan con una actitud bastante así... de disponer.

E: ¿Cómo te sentís relacionándote con ellos?, ¿tenés un contacto cercano, un código común?

P: No, código común no. Creo que ellos están en otro mundo [...] no tengo puntos de contacto, somos muy diferentes.

Frente a esta situación hay posiciones divergentes de los docentes que condensan las operaciones que desarrollan para vincularse y afrontar la distancia que presenta la relación con sus alumnos y familias.
Lo que sigue es una descripción y análisis de las diferentes posiciones identificadas entre los entrevistados. Cabe señalar que se trata de una caracterización de las posturas que hemos podido reconocer a modo de "estilos emocionales" en las prácticas afectivas que presentan los docentes. La noción de estilos emocionales ha sido planteada por Illouz para hacer referencia a "una manera de manejar la relación del yo con los otros y de imaginar sus posibilidades" (Illouz, 2007 , p. 24). En este caso, aludimos a las construcciones emocionales que los docentes efectúan en la medida en que aquello que manifiestan sentir por sus estudiantes no es un sentir espontáneo sino que se encuentra mediado por el ejercicio de su rol.

\section{Los profesores y la instauración de asimetrías en las escuelas privadas}

\section{En el aula se suspenden las diferencias}

Esta posición se ha esbozado por parte de un grupo amplio de los profesores, que sostienen que el aula es un espacio en donde las diferencias y las distancias sociales parecerían esfumarse $\mathrm{o}$, al menos, relativizarse. Son varios profesores de distintas escuelas privadas que formularon que en la escuela "el vínculo se humaniza y todo fluye"; "en el aula instalo mi forma de trabajo, todos son iguales, y podemos interactuar"; 
"más allá de quiénes son sus familias, son chicos de 15 años y en la clase llevamos adelante un espacio de intercambio ameno". Inclusive suelen plantear que hay una diferencia entre las familias y los hijos en el sentido de que la relación cotidiana de los docentes se mantiene con los jóvenes que "son chicos", y esa condición produce una relación que cuenta con la distancia requerida para poder enseñar. Parecería que la condición etaria de los alumnos posibilita un vínculo que supera cualquier diferencia de origen. Como señala una profesora de Arte de un colegio católico:

Ellos en la escuela son mis alumnos, no importa de dónde vienen. Un profesor no se fija en eso, si se fija demasiado, si tuviera resentimiento, no puede enseñar. Ellos vienen a la escuela y a mí me corresponde educarlos, como a cualquier chico.

En este sentido, hay una serie de significados y prácticas que forman parte del legado de la escuela como dispositivo moderno y permiten el vínculo entre los profesores y los alumnos en estos entornos. Entre ellos podemos mencionar: la ubicación de los jóvenes en la posición de alumnos; los niños y los jóvenes considerados sujetos de cuidado y destinatarios del amparo escolar; y la delegación de la autoridad de las familias a las escuelas para la enseñanza formal y la formación de su descendencia. Pese a las transformaciones que acontecen en esta escena moderna (Dubet, 2006), dicha configuración está presente cuando los profesores explican las razones que permiten y facilitan el vínculo pedagógico con estos jóvenes. Esto tiene que ver básicamente con la imposición y la pregnancia de la categoría de "alumnos" por encima de la adscripción social y de origen de sus estudiantes. Las "expectativas de trasfondo" (Garfinkel, 2006), aquello que por corriente y ordinario los actores dan por sentado y que permea la escena escolar, permite el desarrollo de las situaciones de enseñanza en estas aulas.

Algunos docentes que adhieren a esta postura plantean el devenir de un vínculo posible ya sea tanto por la imposición de una autoridad basada en reponer una relación que refuerza las jerarquías ("en el aula respetan mis reglas", dice un profesor de Informática de la escuela privada bilingüe), como por quienes plantean una relación que fluye por la "fuerza del cariño", como sostiene una profesora de Historia de la escuela privada trilingüe. Estos polos antagónicos están muy presentes en varias entrevistas que refieren al establecimiento de los vínculos, ya sea por la fuerza de la jerarquía, o por el afecto que los jóvenes despiertan en los profesores. 
En síntesis, parecería que la condición de tratar con "chicos" desdibujaría el hecho de estar en contacto con los hijos de los poderosos. Al respecto, una profesora de Lengua del colegio católico plantea:

Mucho contacto con las familias no tengo, pero sí con los alumnos como personas. $Y$ entiendo que ellos tienen 15 años, tienen algunas cosas de sus familias, pero otras no. Tengo una buena relación con la mayoría. No tengo mayor problema en relacionarme con gente que no comparte muchas cosas de mi cultura. Nunca tuve ese problema, ni para arriba, ni para abajo. Me engancho con las cosas que compartimos, y las que no compartimos, lo decimos y ya está.

Asimismo, los profesores señalan la diferencia entre ser los "hijos de" y "quiénes son los propios padres", diferencia que hemos registrado habitualmente como una distinción planteada a favor de los alumnos. En este sentido, una profesora de Comunicación del colegio bilingüe señala:

Me cuesta mucho la vinculación con las familias, no con los chicos, me es difícil con los padres y madres. No toleran que los hijos tengan notas bajas, para ellos es un fra- caso, les importa el éxito. Para ellos el éxito no es un proceso, se alcanza mágicamente. Me cuesta porque no puedo entrar en su lógica, que es muy empresarial. Con los chicos me llevo muy bien, sobre todo con los más grandes, los cursos más bajos los dejé porque cuanto más chicos, son el prototipo de eso que ven en sus casas. Cuando crecen, a los 16, que se diferencian de los padres, es muy interesante trabajar con ellos, les vamos abriendo la cabeza.

Estipular esta diferencia clara entre los chicos y sus padres "salva" la relación a favor de los jóvenes. En estas situaciones, los docentes demarcan una clara distinción entre ambos. Convertir a estos jóvenes en estudiantes permite educarlos más allá de sus orígenes. Así, la categoría alumno y el mandato de educarlos se imponen a la condición de origen de las familias; se trata ante todo de educar más allá de quiénes sean los alumnos, en cuanto sujetos en formación. También este acto de educarlos presupone producir cambios que los diferencien de aquellas características de las familias de origen que los docentes impugnan. 


\section{La distancia social neutralizada por la acción institucional}

La mayoría de los profesores alude a las instituciones en las cuales trabajan como entidades que contribuyen a equilibrar las distancias que tienen con las familias de sus alumnos.

Con excepción de la escuela trilingüe, en donde parecería que las autoridades del establecimiento resultan más permeables a las demandas y presiones de las familias, las otras instituciones procesan el diferencial de poder entre el origen y las condiciones de las familias y la labor que deben desarrollar las escuelas, que muchas veces colisiona con algunas de las valoraciones y prácticas de las familias que reciben. Resulta bastante común que los docentes aludan a "adultos que actúan como adolescentes", "padres que discuten las calificaciones obtenidas por sus hijos", "familias íntegras que se van de viaje veinte días en cualquier momento del ciclo escolar sin importarles cómo están sus hijos con el rendimiento de las materias", etc.

En estos casos, las escuelas ofician como intermediarias entre los profesores y las familias. En dos de las escuelas abordadas en nuestro trabajo se destaca especialmente esta tendencia. En primer lugar, los profesores de la escuela católica refieren a esta dinámica mediante la caracterización de la estructura organizacional de la escuela. Señalan que el establecimiento cuenta con una amplia estructura (directores, coordinadores, jefes de departamentos, entre otros) que actúan mediando ante las demandas y los conflictos con las familias y los alumnos.

Yo como docente sólo me entero de algunos de los problemas de mis alumnos a través de los coordinadores o de la directora. En casos excepcionales el docente pide una entrevista. Yo nunca pedí entrevista, un padre o dos en todos estos años me pidieron una entrevista. Mi contacto con lo familiar de los chicos es filtrado por la escuela. (Profesora de Lengua, colegio católico)

En segundo lugar, los docentes del colegio bilingüe aluden a otra modalidad existente para procesar las diferencias que tiene que ver con el prestigio de que goza la institución y que hace que los padres, más allá de su condición de familias muy poderosas, se avengan a las decisiones y normas de procedimiento planteados. Un profesor del colegio bilingüe formula esta cuestión: 
Cuando comencé a trabajar aquí yo tenía algunos prejuicios, pensaba que podía haber chicos demasiado consentidos, con acceso económico y a espacios de poder que yo no tenía. No fue así cuando entré, a medida que fui viendo las cosas, veía que acá el trato es de mucho respeto, son muy pocos los casos en que los chicos sacan esa carta. No existe como instancia. En el colegio se ha apoyado mucho a los profesores en las pocas situaciones que hubo casos así, porque entienden que es un lugar que el colegio tiene que resguardar. Que no se puede permitir, que por la razón que sea, dinero o poder, se avasalle la disciplina del colegio. (Profesor de Física, colegio bilingüe)

En estos casos, hay un consenso entre los profesores acerca de la necesidad de que existan resortes institucionales para afrontar las diferencias de poder que se plantean entre las familias, los alumnos y las escuelas. La distancia que plantea el poder de las familias ante la figura de los profesores resulta en cierta medida suturada y equilibrada a partir de la implantación de un trabajo de orden institucional que ejercen las escuelas para atender a esta asi- metría. Así, las escuelas mediante su trabajo institucional instalan un orden moral (Garfinkel, 2006; Zigon, 2007) y escolar, fundado en la organización institucional o en su trayectoria y prestigio, para intentar aliviar las tensiones que despiertan las distancias sociales existentes entre profesores, familias y estudiantes.

\section{La autenticidad relaja las diferencias}

Otra posición que hemos identificado en el desarrollo de las entrevistas es la de los profesores que, reconociendo las diferencias y distancias de origen en relación con sus estudiantes, consideran que la autenticidad permite sortear cabalmente estas situaciones. Con el término "autenticidad" sintetizamos lo que varios docentes expresan como "blanquear cuál es mi situación", "no querer aparentar lo que no soy y no tengo" (profesora de Literatura, escuela bilingüe). Asimismo, una profesora de Arte del colegio católico plantea que:
[...] no se puede trabajar en estas escuelas si tenés resentimiento hacia los chicos, eso es horrible, si no te sentís cómoda y no podés trabajar con estos alumnos porque crees que vienen de un mundo muy alejado al tuyo, lo mejor que podés hacer es irte. 
Estas posiciones surgen sobre todo asociadas a los consumos ostensibles a los que tienen acceso los estudiantes.

E: ¿Vos te sentís muy lejana al mundo de ellos? P: en algunas cosas, lejanísima. A mi me divierten. Todos los profesores somos pintorescos para ellos. Te cuentan que van a veranear a Punta del Este y yo les digo "yo me fui a Mar del Tuyú, chicos". (Profesora de Historia, colegio trilingüe)

También la autenticidad surge asociada a la valoración que los profesores plantean en relación con las posibilidades materiales que tienen sus alumnos. Esta parecería ser otra manera de mostrarse "auténticos", marcando positivamente la diferencia de origen. En estos casos, los profesores procuran enseñarles a los alumnos a valorar las diferentes experiencias a las que pueden acceder.

El otro día hablaba con una de las chicas que se iba de viaje a Grecia y le decía "cómo te envidio, yo no llego ni a Pinamar". Eso me gusta... festejarles los viajes. "¿Qué viste y qué conociste?" porque está bueno ir a Grecia. "Admiro que puedas más, aprovéchalo", y ahí le comento qué tiene que conocer si o sí en Grecia. (Profesora de Diseño de Imagen, colegio bilingüe)

Ante estas situaciones se registra que los profesores fundan su relación asimétrica a partir de anteponer el capital cultural que portan. En este sentido, los profesores señalan que las familias y sus alumnos tienen cierta admiración por el capital cultural que poseen. Una docente plantea: "los chicos se fascinan, te dicen: 'Uy ¿sabés francés?'". Otra profesora plantea en relación con sus alumnos y los padres: "nos ven como unos bichos raros, extravagantes".

El saber aparece como productor de una mezcla de extrañeza y admiración en un grupo que claramente posee un fuerte poder económico y que reconoce cierta valoración por el contenido cultural, aunque no necesariamente esta sea su apuesta como proyección de futuro en tanto no se trata de las opciones que privilegian en primer lugar estas escuelas. Como señalan varios trabajos que abordan un universo de escuelas y familias semejantes (Gessaghi, 2013; Tiramonti, 2004; Ziegler, 2004), en estos casos la estrategia de reproducción no se centra estrictamente en la posesión de capital cultural como elemento valorado para el 
arribo y mantenimiento de la condición de privilegio y poder de algunos de estos grupos en particular ${ }^{10}$.

Podríamos señalar que la posesión del capital cultural permite demarcar una diferencia de poder de los profesores con respecto a las familias y sobre todo sus alumnos. Esta diferencia permite reponer la asimetría que hace posible un vínculo con la distancia necesaria para facilitar la transmisión pedagógica. Ante la distancia que impone la diferencia de origen económico, como dice un docente de informática "yo soy pobre, al lado de estos [los alumnos y sus familias] cualquiera es pobre". El capital cultural que los profesores poseen permite reponer la distancia requerida para enseñar y relajar la tensión que plantean las diferencias, en la medida en que todos detentan algún tipo de capital que se pone en juego en dicha relación.

De acuerdo a la planteado hasta aquí cobra evidencia que la posesión de un quantum de capital diferencial a favor de los profesores resulta una condición sine qua non para poder entablar el vínculo pedagógico, pero dicha diferencia debería ser reconocida por ambas partes, o al menos, pese a la distancia deberían existir determinados códigos en común o vasos comunicantes entre las experiencias de todos para que la relación

10 En Tiramonti (2004) y Ziegler (2004) puede advertirse que este tipo de escuelas son las que priorizan la formación para entornos cosmopolitas y altamente competitivos, y no se pone especial énfasis en la formación humanista y científica más clásica, como en otros establecimientos. resulte posible. En este sentido, la autenticidad se despliega cuando ambas partes, a partir de sus diferencias, echan a rodar el vínculo que supone toda relación pedagógica.

\section{Abordar las diferencias mediante los contenidos escolares}

La posición que plantearemos aquí también está presente en las mismas escuelas caracterizadas en el punto anterior (bilingüe y trilingüe). En una serie de entrevistas, los profesores plantearon que el hecho de que su labor consiste en el tratamiento del conocimiento, la situación de enseñanza permite abordar las diferencias sociales y problematizar las posiciones de élite que eventualmente ocuparán sus estudiantes. Este sería entonces un tema por abordar desde el contenido cultural que se trata en las clases. Estos profesores vislumbran las posibilidades transformativas que proponen ejercer sobre sus alumnos. En otros casos, tal vez no haya una voluntad de transformación tan radical pero los docentes plantean la necesidad de que la experiencia cotidiana de sus alumnos resulte problematizada y no naturalizada.

E: ¿Cómo es el contacto tuyo con las familias, te sentís muy distante? ¿Qué implica ser profesora de estos chicos? 
P: Que mi mundo no tiene nada que ver con el de los chicos, seguro. Tampoco siento que eso me ponga en una posición superior o inferior. Sí me parece que en el momento que estoy frente al aula, hay determinadas preguntas, determinadas respuestas de los chicos, o comentarios, que sé que no tengo que hacer, que no puedo hacer, o no tiene sentido que haga. Cuando me paro delante del aula tengo presente que son chicos, te lo digo así, que tienen más plata, y que tienen un origen socioeconómico distinto del mío. Pero no sé, soy consciente de eso. No sé como decírtelo. Lo tengo bien presente, no creo que se modifique mi enseñanza, sí quizás cuando elijo los textos, voy pensando en esas diferencias. Al mismo tiempo a veces trato de exponerlos a situaciones distintas a esas que ellos viven. No te dije nada claro.

E: Sí. Cuando elegís un texto, ¿en qué sentido tenés en cuenta quiénes son ellos?

P: Tampoco es siempre, a veces. Me parece que pensar un poco a veces, no sé si en el contenido, ¡ojo! no se trata de hacerlos leer cuentos de chicos pobres. No pasa por ahí. Sí textos que los hagan pensar que el contexto sociocultural al que una persona pertenece muchas veces determina su forma de actuar. Y que eso se puede dar en un ambiente de pobreza, o en cualquier otro medio. Quizás enfocarlo por ese lado. Eso. Busco que piensen y trasciendan un poco más, que no todo es la burbuja en donde ellos viven. Que también hay burbuja en la villa. Tratar de comprender, no ponerlo en términos de riqueza o pobreza, sino de que cada uno está rodeado por determinados parámetros y una realidad, y tiende a pensar que eso es lo único, y hay que pensar más allá. (Profesora de Literatura, colegio bilingüe)

Un profesor de Filosofía del colegio trilingue señala en un sentido semejante:

Hay temas de mi materia que funcionan muy bien: cuando los planteos filosóficos tienden más a un planteo crítico e individual, a la autonomía de pensamiento. Todo aquello que si querés supone que los chicos puedan cuestionar todo y sentir el poder de su pensamiento, en eso los pibes son bárbaros. Juegan. 
A todos los vuelvo nietzcheanos. Esta cosa de desconfiar de todo, todo puesto en duda. Cuando se trata después de construir una comunidad, ahí falla más. En todos los grupos tenés aquellos chicos más encolumnados en una postura liberal-meritocrática. Con lo cual, se dan buenas polémicas. Yo discuto mucho con ellos en clase. Doy Filosofía de la Economía, Historia del Pensamiento Económico. Doy Marxismo. Vienen de la escuela de negocios, vienen preparándose para el management empresarial y se encuentran con esta materia donde tienen que vérselas con aquello que se supone los sostiene en ese lugar. Cuando intentan defender sus posturas a través del diálogo, siguen sosteniéndolas, pero por lo menos las fundamentan. No es lo mismo que digan que tienen lo que tienen porque lo merecen, después de esta materia se dan cuenta, que no pueden decir eso, así nomás... esa vuelta funciona. (Profesor de Filosofía, colegio trilingüe)
En las palabras de estos docentes no se registra esta tendencia a saldar las diferencias con sus alumnos por la vía de la autenticidad o por la vivencia del aula como un espacio en donde las distancias se borran o al menos se relajan, sino que precisamente el poder, el privilegio y las condiciones de vida de diferentes grupos sociales resultan objeto de trabajo en la labor de transmisión. En el caso de ambos profesores el conocimiento resulta la llave que permite el vínculo y el cuestionamiento de las situaciones de poder diferencial, ya sea tanto en el contexto del aula como fuera de ella. Así, la formación de las élites en cuanto tales (Howard, 2010) tiene lugar mediante la desnaturalización de la propia condición a través de la enseñanza de los contenidos del curriculum escolar.

\section{Las asmiterías en la escuela pública: la distancia social y cultural disputada}

En el caso de la institución de dependencia universitaria es factible reconocer ciertas dinámicas que se desarrollan para procesar las diferencias sociales y culturales entre profesores y estudiantes, que merecen un tratamiento por cuanto constituyen otro modo en que se exhiben y canalizan las diferencias en los terrenos social y cultural.

En oposición a los casos anteriores, en la institución estatal los profesores no perciben una distancia tan radical con respecto a ellos y sus 
estudiantes en términos de origen y trayectorias sociales. Aquí, el modo de reclutamiento de los alumnos estaría dando lugar a una visión de un grupo de estudiantes relativamente heterogéneo en la medida en que comprende procedencias sociales más variadas (Méndez, 2013). En este contexto, los profesores reconocen un universo de ideas, aspiraciones y valoraciones compartidas. Un profesor de Física decía "yo podría haber sido uno de estos pibes" (haciendo alusión a sus alumnos), una profesora de Lengua plantea en un sentido semejante: "me siento identificada con los chicos de acá, me siento identificada con lo que fue mi adolescencia". Estas identificaciones no han surgido en los profesores de las otras escuelas, donde prima la distancia y ajenidad con las vivencias de los estudiantes.

Ante esta condición de mayor homogeneidad social y cultural entre profesores y estudiantes, lo que advertimos con frecuencia en las entrevistas es la disputa que plantean los estudiantes hacia los profesores como modo de vinculación habitual.

Cuando los profesores de esta institución se refieren al trato con sus estudiantes suelen destacar que, si bien no se trata de alumnos indisciplinados, es común que sean soberbios y traten con desdén a los docentes. En este sentido, no se trata de la constatación de una imagen de alumnos que requieren el cuidado de los adultos sino que, por el contrario, aluden a jóvenes que ponen a prueba las condiciones de sus profesores y que viven el cotidiano escolar en una institución donde se cuestionan fuertemente las distancias, jerarquías y la autoridad. Ante la imposición de esta jerarquía los profesores destacan episiodos en los que se producen "disrupciones de la actuación" (Goffman, 1989), donde se trastocan las relaciones que se dan por sentadas en los vínculos entre profesores y estudiantes.

En cuanto a la arrogancia de los estudiantes, un profesor de Biología advierte: "El hecho de la libertad que les damos de participación a veces los transforma en monstruitos, intolerantes, soberbios. [...] A veces realmente creen que pueden opinar a la par del Rector. Les cuesta entender la asimetría entre adulto-adolescente".

En las entrevistas hay variados testimonios que dan cuenta de esta tensión y de disputas entre los profesores y estudiantes.

Por una parte, algunos entrevistados refieren a los vínculos jerárquicos que la institución sostiene. Las referencias edilicias son un indicador de orden material y objetivo que emplean para apoyar estos argumentos: 
Creo que la institución misma, al ingresar, no solo por la trayectoria, sino por la estructura material del colegio hace que uno se sienta parte de algo enorme y que existe hace muchos años. Y que, como les digo a los alumnos, que no se haya modificado su estructura estética, no es casualidad. La permanencia de lo antiguo, se da así porque acá vale mucho la tradición. Si durante estos años el colegio produjo tanta cantidad de personajes notables, la estructura no debe ser tan mala. La sala de profesores no es un cuartito como en otros lugares. Acá es un lugar espacioso. La directora no está en una silla de plástico. En el aula uno ve que el profesor está ubicado sobre dos escalones por encima de los alumnos. Cuando el alumno ve eso, más lo que se recalca, se da cuenta de que la simetría en este colegio entre alumno y docente no funciona. El docente está dos escalones por encima, no es por autoritarismo sino por sabiduría. Los que más saben enseñan a los que menos saben. Este discurso está permanentemente acá. Acá todavía el profesor tiene reconocimiento. (Profesora de Historia)
Por otra parte, algunos profesores señalan la impugnación y la puesta a prueba que los alumnos efectúan a sus docentes de modo que demuestren que poseen la gallardía necesaria para ocupar ese lugar, plantean el desafío a los profesores que deben demostrar que están "a la altura de las circunstancias" para formarlos. Así, "los que más saben" deben constatarlo ante el escrutinio explícito de su discípulos.

$$
\begin{aligned}
& \text { Cuando yo ingresé como } \\
& \text { profesora hace unos años } \\
& \text { entré a cargo de un curso } \\
& \text { en reemplazo del jefe de } \\
& \text { departamento, me tocó a } \\
& \text { mí. Yo era más joven. No } \\
& \text { era la jefa, había estudiado } \\
& \text { en el profesorado, no era } \\
& \text { universitaria y me pregun- } \\
& \text { taron "¿Ud. qué estudió?". } \\
& \text { Cuando les dije que era } \\
& \text { profesora, me dijeron } \\
& \text { "igual es grosa". Como } \\
& \text { que pese a ese título reco- } \\
& \text { nocían que sabía mucho. } \\
& \text { Hablaba fluidamente de } \\
& \text { mi materia y de otros } \\
& \text { temas porque siempre fui } \\
& \text { muy lectora, entonces los } \\
& \text { chicos decían "esta sabe, } \\
& \text { tiene opinión". (Profesora } \\
& \text { de Química) }
\end{aligned}
$$

Si los docentes son quienes definen la trayectoria y promoción de los alumnos, en esta institución estos últimos se arrogan poner a prueba la idoneidad de estos profesores para ocupar dicho lugar. En este sentido, si los profesores son los operadores a 
cargo de la selección y consagración que efectúa este establecimiento, los alumnos los someten a testeo para certificar su cabal capacidad.

Los vínculos en el colegio universitario se caracterizan por una mayor distancia y resultan una esfera inclusive de confrontación entre los jóvenes y los adultos. En el marco del denominado "declive de las instituciones" (Dubet \& Martuccelli, 2000) en esta institución parecería haber una tendencia a sostener la distancia y la imposición de la autoridad antes que a dar lugar al avance de las relaciones interpersonales y la proximidad. La apelación y el sostenimiento de una autoridad impuesta por la distribución formal de las posiciones de poder procura mantener al margen a los vínculos de carácter más cercano. Si en términos de estos autores cada vez más las relaciones pedagógicas debilitan el rol en beneficio de la relación, en este caso parecería que hay un esfuerzo por resistir a los embates de dicho declive sosteniendo las formas vinculares entre adultos y jóvenes que caracterizaron los tiempos pretéritos.

\section{Conclusiones}

A lo largo de estas páginas, hemos desarrollado los modos en que se vehiculizan y dirimen las distancias que se presentan entre profesores y estudiantes cuando estos últimos provienen de medios sociales más favorecidos con respecto a los de los profesores o cuando los profesores se encuentran ante alumnos que tal vez pertenezcan a un medio social y cultural semejante y aspiran a ocupar posiciones de élite.

En estos enclaves las relaciones asimétricas extraescolares que empoderan a los estudiantes son sustituidas, asumiendo los docentes una posición que tiende a reemplazar la situación de poder que portan los alumnos y reubicar un vínculo de otro orden con ellos. Este trabajo y accionar de los profesores en pos de invertir las relaciones de poder que tienen con las familias por fuera del escenario escolar se efectúa para instaurar una asimetría que constituye una condición sine qua non requerida para la enseñanza.

Nuestro estudio da cuenta de los diferentes mecanismos puestos en juego por los profesores en el momento de generar asimetrías para erigirse como autoridad pedagógica. En este sentido identificamos diferencias sustantivas entre las escuelas que privilegian la posesión de recursos económicos familiares como criterio excluyente para el acceso a los establecimientos 
(escuelas privadas que reclutan a las familias de elevados ingresos), y la institución universitaria que se rige por un criterio de reclutamiento cuya forma de selección de los estudiantes y su legitimación en el acceso a ese espacio exclusivo es meritocrática (escuela pública y gratuita dependiente de la universidad).

En las escuelas privadas donde el mecanismo de reclutamiento se produce con base en el poder económico de las familias advertimos una mayor presencia de la labor desarrollada por los profesores a los efectos de invertir en el aula las relaciones de poder existentes entre los docentes y las familias por fuera de la escuela. Los mecanismos institucionales empleados se desarrollan para que estos profesores puedan entablar una relación asimétrica frente a los hijos de los poderosos. En el caso de la escuela pública universitaria se advierte una extensa tradición e imposición de mecanismos institucionales que refuerzan la presencia de una jerarquía diferencial entre profesores y estudiantes; jerarquías que se destacan en los vínculos y la propia arquitectura escolar. En este ámbito se advierten fuertes disputas entre los profesores y estudiantes, en un ambiente en el cual los primeros deben demostrar ante sus alumnos su íntegra capacidad y gallardía para formar a las futuras élites. El trabajo de campo da cuenta de los modos en que se constituye la asimetría y las relaciones de autoridad entre profesores y alumnos en un contexto de escuelas de élite que se encuentra interiormente fragmentado en virtud de la población que tendencialmente incorpora cada uno de los perfiles de establecimientos en particular.

Los profesores de estas escuelas intervienen creando relaciones de poder diferencial. En los registros de nuestras entrevistas se observa que dichas relaciones procuran restituir vínculos de autoridad con sus estudiantes de carácter semejante a los del proyecto escolar moderno: una autoridad basada en el saber y la jerarquía entablada por los profesores frente a los estudiantes como generación adulta a cargo de la transmisión de los saberes a la generación joven. Si bien pueden presentarse disputas o impugnaciones ante la autoridad de los profesores, se registra un esfuerzo de estos y de las instituciones por afrontar los embates que atraviesa la matriz de la escuela moderna. En estos espacios, la constitución de una asimetría y de relaciones de autoridad se postula como una condición sine qua non para entablar los vínculos pedagógicos. En virtud de ello, los docentes despliegan los mecanismos de los que hemos dado cuenta a lo largo de las páginas precedentes. 


\section{Referencias bibliográficas}

Abramovsky, A. (2010). Maneras de querer. Los afectos docentes en las relaciones pedagógicas. Buenos Aires: Paidós.

Antelo, E. (2007). Alarma en las escuelas. Miedo, seguridad y pedagogía. Revista Propuesta Educativa, 27, 7-17.

Darmon, M. (2013). Les classes préparatoires: fabrique d'une jeunesse dominante. Paris: Editions La Découverte.

Daverne, C. \& Dutrecq, Y. (2013). Les bons élèves. Expériences et cadres de formation. Paris: PuF.

Dubet, F. (2006). El declive de la institución. Profesiones, sujetos e individuos en la modernidad. Barcelona: Gedisa.

Dubet, F. \& Martucceli, D. (2000). ¿En qué sociedad vivimos? Buenos Aires: Losada.

Duschatzky, S. \& Redondo, P. (2000). Las marcas del Plan Social Educativo o los indicios de ruptura de las políticas públicas. En S. Duschatzky (comp.) Tutelados y asistidos, programas sociales, políticas públicas y subjetividad. Buenos Aires: Paidós.

Dussel, I. \& Caruso, M. (1999). La invención del aula. Una genealogía de las formas de enseñar. Buenos Aires: Santillana, Buenos Aires.

Garfinkel, H. (2006). Estudios en etnometodología. Barcelona: Anthropos.

Gessaghi, V. (2013). Familias y escuelas: construcción del sentido de la educación y de la escolarización en "la clase alta argentina". RUNA, Revista del Instituto de Ciencias Antropológicas, 34 (1), 73-90.

Goffman, E. (1989). La presentación de la persona en la vida cotidiana. Buenos Aires: Amorrortu.

Howard, A. (2010). Stepping outside class: afluent students resisting privilege. En A. Howard \& R. A. Gaztambide-Fernández, Educating elites. Class privilege and educational advantage. Maryland: R\&L Education.

Illouz, E. (2007). Intimidades congeladas. Las emociones en el capitalismo. Madrid: Katz.

Kahn, S. R. (2011). Privilege. The making of an adolescent elite at St. Paul's School. Nueva Jersey: Princeton University Press.

Kessler, G. (2002). La experiencia escolar fragmentada. Estudiantes y docentes en la escuela media de Buenos Aires. Buenos Aires: ॥PE-Unesco. 
Kòjeve, A. (2005). La noción de autoridad. Buenos Aires: Nueva Visión.

Martinis, P. \& Redondo, P. (2006). Igualdad y educación: escrituras entre dos orillas. Buenos Aires: Del Estante Editorial.

Méndez, A. (2013). El colegio. La formación de una élite meritocrática en el Nacional Buenos Aires. Buenos Aires: Sudamericana.

Narodowski, M. (2014). Infancia, pasado y nostalgia: cambios en la transmisión intergeneracional. Revista Brasilera de Historia de la Educación, Maringá-PR, 14 (35), 191-214.

Rauscher, J. B. (2010). Les professeurs des classes préparatoires aux grandes écoles: une élite au service des élites. Tesis doctoral. Observatoire Sociologique du Changement, Sciences Po, Francia, mimeo.

Rodríguez Moyano, I. (2012). Capital cultural y estrategias educativas de las clases altas de la Ciudad de Buenos Aires. En: S. Ziegler \& V. Gessaghi (comp). Formación de las élites. Investigaciones y debates en Argentina, Brasil y Francia. Buenos Aires: Manantial.

Tiramonti, G. (2004). La fragmentación educativa y los cambios en los factores de estratificación. En: G. Tiramonti (comp.), La trama de la desigualdad educativa. Mutaciones recientes de la escuela media. Buenos Aires: Manantial.
Tiramonti, G. \& Ziegler, S. (2008). La educación de las élites. Aspiraciones, estrategias y oportunidades. Buenos Aires: Paidós.

Weber, M. (1992). Economía y Sociedad. México: FCE.

Ziegler, S. (2004). La escolarización de las élites: un acercamiento a la socialización de los jóvenes de sectores favorecidos en la Argentina actual. En G. Tiramonti (comp.), La trama de la desigualdad educativa. Mutaciones recientes de la escuela media. Buenos Aires: Manantial.

Zigon, J. (2007). Moral breakdown and the ethical demand. A theoretical framework for an anthropology of moralities. Anthropological Theory, 2 (7), 131-150. 